\title{
Annular Beam Shaping in Multiphoton Microscopy to Reduce Out-of-Focus Background
}

\author{
Johan Borglin,, ${ }^{1,2}$ Danni Wang, ${ }^{1}$ Nicholas J. Durr, ${ }^{3}$ Dag Hanstorp, \\ Adela Ben-Yakar, ${ }^{4}$ and Marica B. Ericson ${ }^{1}$ \\ ${ }^{1}$ Biomedical Photonics Group, Department of Chemistry and Molecular Biology, University of Gothenburg, Kemivägen 10, \\ 41296 Gothenburg, Sweden \\ ${ }^{2}$ Department of Physics, University of Gothenburg, Kemivägen 9, 41296 Gothenburg, Sweden \\ ${ }^{3}$ Department of Biomedical Engineering, Johns Hopkins University, 3400 N. Charles St., Baltimore, MD 21218, USA \\ ${ }^{4}$ Department of Mechanical Engineering, University of Texas, 204 E. Dean Keeton Street, Austin, TX 78712, USA
}

Correspondence should be addressed to Marica B. Ericson; marica.ericson@chem.gu.se

Received 19 January 2017; Accepted 15 March 2017; Published 24 May 2017

Academic Editor: Kam-Sing Wong

Copyright (C) 2017 Johan Borglin et al. This is an open access article distributed under the Creative Commons Attribution License, which permits unrestricted use, distribution, and reproduction in any medium, provided the original work is properly cited.

Despite the inherent spatial confinement of multiphoton processes that arises from focusing through an objective, the maximum imaging depth in conventional multiphoton microscopy is ultimately limited by noise from out-of-focus fluorescence. This is particularly evident when imaging beyond shallow depths in highly scattering tissue as increased laser powers are necessary. The out-of-focus signal originates from multiphoton processes taking place primarily at shallow depths and deteriorates contrast and limits imaging depth. In this paper, annular laser beams are explored as a concept to reduce this background signal in multiphoton microscopy. The approach is theoretically verified by data from simulations and proof of principle is demonstrated on a custom-built experimental multiphoton microscopy platform. Annular laser beams were created by adopting wavefront control using a spatial light modulator and implemented for imaging tissue phantoms simulating turbid media and human skin ex vivo. The signal-tobackground ratios were calculated and compared to images acquired with a traditional, filled-aperture Gaussian beam. Experiments in tissue phantom show an improvement in signal-to-background ratio of about $30 \%$ when using annular beam illumination in comparison to Gaussian illumination at specific depths. When laser power is not the limiting factor, this approach is expected to provide even greater benefits.

\section{Introduction}

Multiphoton microscopy (MPM) has evolved from a photonic novelty [1] to a well-established laboratory tool that allows for noninvasive 3D imaging of tissue [2]. Since MPM is operating in the "optical window" of biological tissue $(\lambda \sim 600-1300 \mathrm{~nm})$, it allows for increased imaging depths compared to single photon excitation modalities like confocal microscopy [3]. MPM utilizes high intensity fs-pulse lasers to generate a high flux of photons necessary for multiphoton absorption. Conventionally, these nonlinear processes are assumed to occur only at the focal volume where extremely high photon flux is generated, allowing for noninvasive threedimensional optical sectioning. Recently, MPM has enabled powerful applications in life sciences such as high-speed cell mitosis imaging [4], real-time lymphocyte tracking [5], and cell migration monitoring [6]. MPM has also been established as a tool for visualization in turbid biological matter such as the human skin $[7,8]$. For dermatological purposes, multiphoton microscopy has been commercialized for clinical use (DermaInspect, Jenlab, Germany) [9]. In addition, in vivo MPM is advancing the field of neuroscience by allowing for optical visualization of neural architecture and functions $[10,11]$.

Assuming sufficient excitation power and aberration control, the fundamental factor limiting imaging depth when performing MPM in tissue is the background fluorescence originating from out-of-focus areas above the imaging 


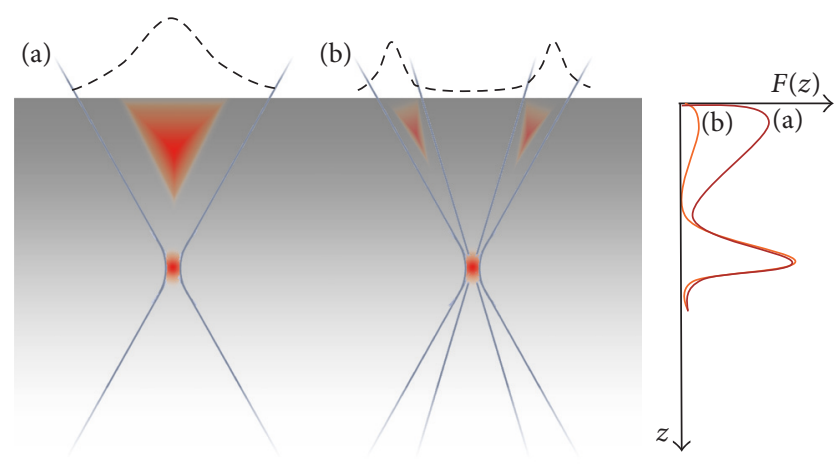

FIGURE 1: Schematic illustration of the concept of annular laser beam to reduce out-of-focus signal in MPM for the two conditions (a) where Gaussian beam profile is implemented and (b) for annular beam illumination. The figure illustrates the hypothetical relative contribution of fluorescence signal, $F(z)$, as a function of depth, $z$. While comparing conditions given equal photon fluences in the focal region, the Gaussian illumination (a) is expected to generate elevated levels of out-of-focus signal from the incoming planes where the laser power is at a maximum compared to annular illumination (b).

plane [12-14]. The out-of-focus signal is a result of increasingly high excitation powers that are necessary to maintain high excitation intensity when the focal volume is located deep in the tissue. This background signal deteriorates the imaging contrast (signal-to-background ratio) and in turn limits imaging depth. Thus, efforts should be made to minimize this undesired signal, to improve the signal-tobackground ratio and thereby increase the imaging depth. The implementation of a spatial filter, such as a confocal pinhole, should allow for blocking the out-of-focus signal; however, the decreased collection efficiency deems this approach suboptimal in MPM as previously discussed [14]. Instead, we here propose an approach based on annular beam shaping. As illustrated in Figure 1, the fluorescence, $F$, generated at a plane, $z$, would be more confined to the focal plane when using an annular laser beam when compared to Gaussian beam illumination. Geometrically, the peak irradiance of the laser light at the sample surface can be lowered by distributing the laser energy in a ring instead of a Gaussian beam profile. This lowers the intensity in any given point of the sample while retaining the photon flux in the focal volume and thus reduces the generation of out-of-focus fluorescence contributing to the undesired background. Previously, annular beam shaping has been investigated for confocal microscopy [15] and multiphoton microscopy $[16,17]$ to improve resolution. The concept of using annular beams to reduce out-of-focus signal is previously not explored.

This study investigates the potential of annular beams to improve signal-to-background ratio in MPM. The implementation of annular beams in optical microscopy gives an elongated focal volume, which results in beam profiles similar to that of a Bessel beam. Therefore, when explored for MPM, the potential effect on the focal volume should be considered. This paper investigates the benefits and tradeoffs of using annular beams by combining theoretical simulations and proof-of-concept experiments using wavefront controlling optics. The work is an extension of our previous approach [18]. Here, a spatial light modulator (SLM) was introduced in the optical path of a custom-built experimental MPM platform in order to obtain an annular beam, similar to the approach shown by Nie et al. [19]. By changing the pattern on the SLM, a systematic comparison of annular versus Gaussian illumination could be performed. Fluorescent scattering tissue phantoms simulating the properties of optically dense biological tissue were investigated, together with excised human skin tissue in order to demonstrate proof of concept.

\section{Materials and Methods}

2.1. Theoretical Simulations. In order to simulate the excitation intensity generated in the focal region when switching from Gaussian to annular beam shape, the Fresnel-Kirchhoff integral was calculated for varying beam shape conditions, based on a previously described approach [18, 20]. The Fresnel-Kirchhoff integral is stated as

$$
\Psi_{\mathrm{FK}}^{\prime}\left(r^{\prime}, \theta^{\prime}, z\right)=\frac{2 \pi}{z \lambda} \int \Psi^{\prime}(r, \theta) e^{(-2 \pi i R / \lambda)} \Phi_{l} d S,
$$

corresponding to the total field amplitude in a point $\left(r^{\prime}, \theta^{\prime}\right)$, at the distance $z$ from the lens. The coordinates are schematically illustrated in Figure 2. Here, $\Psi^{\prime}(r, \theta)$ is the field amplitude at a point $(r, \theta)$ on the lens $(z=0)$. The integration is made by summing over the contributions from each area element, $d S$, on the lens surface. The phase retardation of the lens, $\Phi_{l}$, is given by

$$
\Phi_{l}=e^{i \pi r^{2} / \lambda f}
$$

Here, $f$ is the focal length of the lens, $\lambda$ is the wavelength, and $R$ is the distance vector from the point $(r, \theta)$ on the lens to the point $\left(r^{\prime}, \theta^{\prime}, z\right)$ for which the total field, $\Psi_{\mathrm{FK}}^{\prime}\left(r^{\prime}, \theta^{\prime}, z\right)$, is calculated using the expression

$$
R \approx z+\frac{r^{2}+r^{\prime 2}-2 r r^{\prime} \cos \left(\theta-\theta^{\prime}\right)}{2 z^{\prime}} .
$$

The field amplitude at the lens $\Psi^{\prime}(r, \theta)$ was given by the expression

$$
\Psi^{\prime}(r, \theta)=\frac{P_{0}}{\pi B^{2}}\left(1-\frac{b}{r}\right) e^{-2((r-b) / B)^{2}},
$$

where $P_{0}$ is the laser power at the lens, $B$ is the outer radius of the beam, and $b$ is the inner radius of the beam. The outer radius $B$ was scaled down with increasing $b / B$ to ensure that the total energy was kept constant in the comparisons between different conditions. The Fresnel-Kirchhoff integral (1) was calculated using MATLAB (MathWorks, Natick, MA, USA) for a grid around the focus, in order to simulate the intensity distribution around the focal volume. Each beam shape was characterized by the ratio between the inner radius $(b)$ and outer radius $(B)$, ranging from $b / B=0$ for 


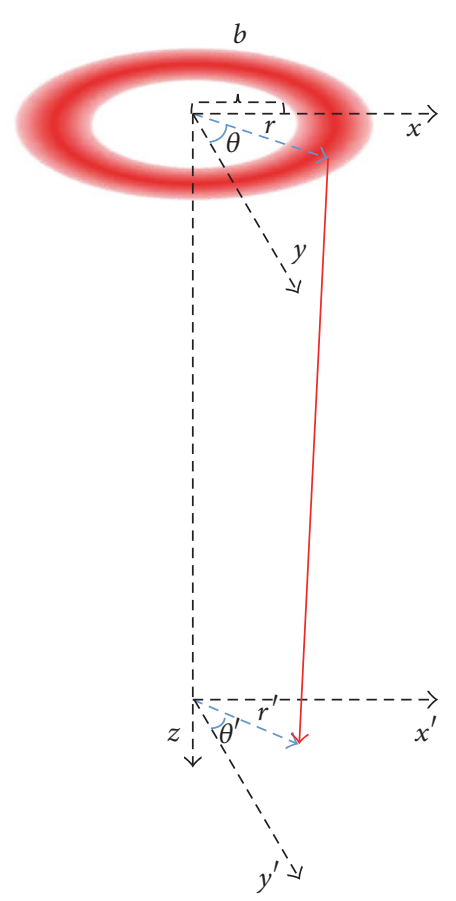

(a)

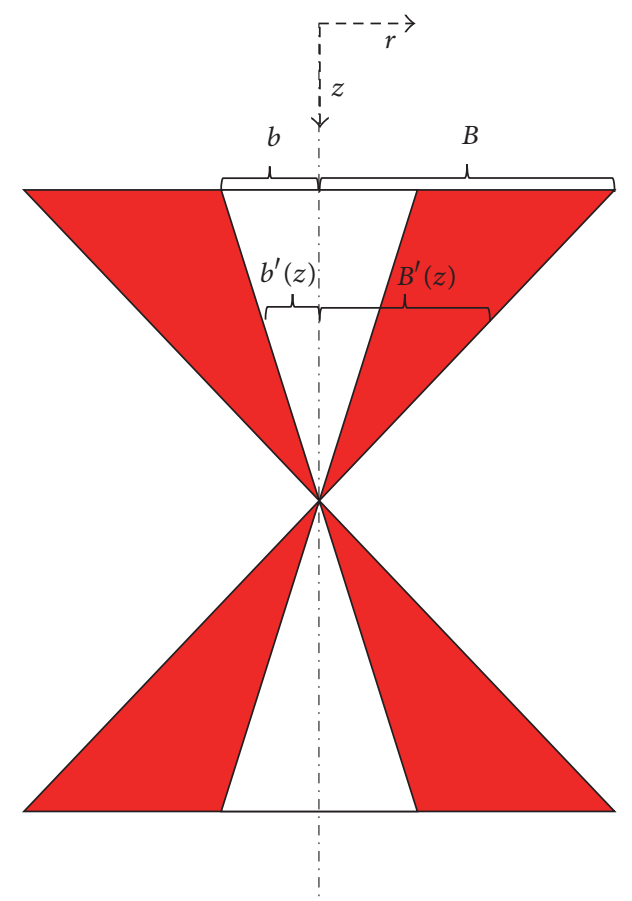

(b)

FIGURE 2: Schematic view of the coordinates for theoretical simulations; (a) the coordinates used for calculating the Fresnel-Kirchhoff integral. For every point $\left(r^{\prime}, \theta^{\prime}, z\right)$ located at a distance $R$ from the lens, the contribution from each element $(r, \theta)$ on the lens $(z=0)$ is added; (b) coordinates for considering the out-of-focus signal generated adopting simplified geometrical focusing conditions.

Gaussian illumination up to the extreme annular case with $b / B$ approaching 1 .

In addition to exploring the effects on the focal volume, the expected generation of out-of-focus fluorescence was calculated by a geometric approach, using the coordinates as illustrated in Figure 2(b). This is a simplified model that assumes that only the ballistic photons are responsible for generating two-photon excitation. Though the scattered semiballistic photons also contribute to out-of-focus fluorescence [12], this approach was considered to be a conceptually simple and valid model to estimate the potential reduction of out-of-focus fluorescence in relative terms. The field amplitude $\Psi^{\prime}(z)$ was calculated as a function of $z$, where the Gaussian beam profile is given by

$$
\Psi^{\prime}(z)=\frac{P_{0}}{\pi B^{\prime 2}} e^{-2\left(r^{\prime} / B^{\prime}\right)^{2}}
$$

and the annular beam is given as

$$
\Psi^{\prime}(z)=\frac{P_{0}}{\pi B^{\prime 2}}\left(1-\frac{b^{\prime}}{r^{\prime}}\right) e^{-2\left(\left(r^{\prime}-b^{\prime}\right) / B^{\prime}\right)^{2}}
$$

$P_{0}$ is the peak power at the exit of the lens, $r^{\prime}$ is the radial coordinate, and $B^{\prime}$ and $b^{\prime}$ are the beam width and inner beam radius, respectively, which decrease as a function of depth $z$ through the relation according to

$$
\begin{aligned}
& B^{\prime}(z)=B\left(1-\frac{z \tan \alpha}{a_{0}}\right), \\
& b^{\prime}(z)=b\left(1-\frac{z \tan \alpha}{a_{0}}\right) .
\end{aligned}
$$

$B$ and $b$ are the beam width and inner radius at the aperture exit, $a_{0}$ is the aperture radius, and $\alpha$ is calculated from the NA, described by

$$
\mathrm{NA}=n \sin \alpha \approx n \frac{D}{2 f} .
$$

The generated out-of-focus fluorescence was estimated to be proportional to the square of the field intensity at the varying $z$-levels, that is, at different depths, so that the total background fluorescence was calculated as

$$
F_{B}=\int \Psi^{\prime}(z)^{2} e^{-2 z \mu_{s}} d z .
$$

The attenuation term in (9) was included to account for attenuation of both excitation light and emitted fluorescence in the sample. The value of the scattering coefficient $\mu_{s}$ was set to $150 \mathrm{~cm}^{-1}$, that is, corresponding to approximate parameters of human skin [21-23]. The value of $F_{B}$ was calculated for a Gaussian beam condition and annular beams 


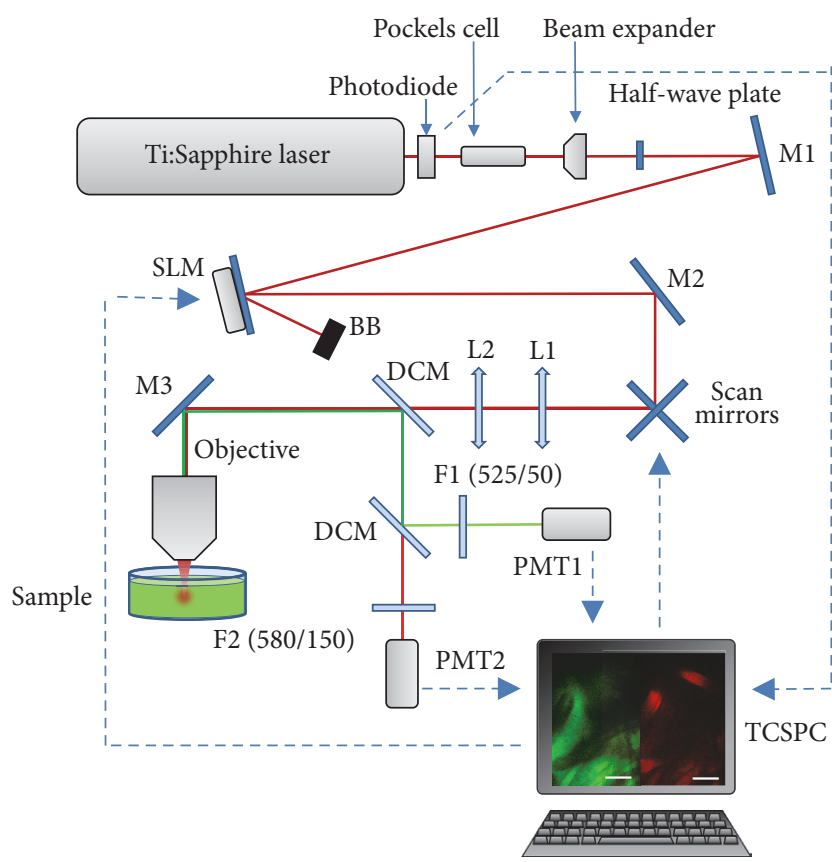

FIGURE 3: Schematic illustration of the experimental MPM platform. We introduced an SLM in the excitation path to form an annulus at the back aperture of the objective. M: mirror. L: lens. F: filter. SLM: spatial light modulator. BB: beam blocker. DCM: dichroic mirror. PMT: photomultiplier tube. TCSPC: time-correlated single photon counting data acquisition.

with a range of $b / B$ values. The implemented parameters used for the simulations were chosen to correspond to the experimental setup, with numerical aperture of the objective $\mathrm{NA}=0.8$, beam waist at the aperture exit $B=1.4 \mathrm{~mm}$, water immersion with refractive index $n=1.33$, and laser wavelength $\lambda=800 \mathrm{~nm}$. The distribution of fluorophores within the sample is assumed to be homogenous. The fluorescence signal was calculated as being proportional to the light intensity square dependence as valid for two-photon excitation processes. The probability of collecting the emitted photons was adjusted by multiplying by different functions for simulating various collection efficiencies. Both flat and Gaussian shaped collection efficiencies were investigated. From these simulations, values of the fluorescence generated within the focal volume $\left(F_{f}\right)$ and outside of the focal volume $\left(F_{B}\right)$ were computed based on the square dependence of the light intensity to calculate signal-to-background ratios (SBR) defined as

$$
\mathrm{SBR}=\frac{F_{f}}{F_{B}} .
$$

Thus, in order to maximize the value of SBR, the value of $F_{B}$ should be kept at a minimum.

2.2. Experimental Multiphoton Setup. Experimental measurements were performed using a custom-built MPM platform schematically illustrated in Figure 3. An SLM (X1046802 LCOS-SLM, Hamamatsu Inc.) was used for wavefront control and beam modulation. The annular beam was created by shifting the phase of the central part of the beam using a triangle wave pattern, thus diverting the central portion away from the beam path. The shape of the annular beam was visualized using a beam profiler (Ophir-Spiricon SP90281). A Ti:Sapphire mode locked laser (Tsunami, Spectra-Physics) was used as the excitation source. The laser is tunable in the wavelength range $700-1050 \mathrm{~nm}$ and provides a repetition rate of $80 \mathrm{MHz}$ with pulse duration of $\sim 100 \mathrm{fs}$. A pump power of $6 \mathrm{~W}$ results in an average laser beam energy of $800 \mathrm{~mW}$ as the beam exits the laser cavity. Laser power was controlled using a Pockels cell (350-80LA, Conoptics). A beam expander (BE05M-B, Thorlabs) was implemented to ensure utilization of the full SLM area and fill the back aperture of the focusing objective. A water-immersion objective (40x, NA 0.8, cover glass correction, Achroplan NIR, Carl Zeiss) was used and mounted on a Zeiss Axiovert in an inverted configuration. The sample was positioned using a combination of manual control for the objective height and a piezoelectric stage (MicroStage/NanoZL500, Mad City Labs) controllable through a LabVIEW program (National Instruments). Data acquisition was performed using two time-correlated single photon counting cards (TCSPC, SPC-150, Becker \& Hickl), here operating in intensity mode.

2.3. Tissue Mimicking Fluorescent Phantoms. Fluorescent phantoms simulating the optical properties of tissue were prepared by dissolving $1 \%$ weight fraction of agar (VWR, PN: 20767) in 1x PBS (pH 7.4, Sigma, PN: P4417) and adding fluorescein (Sigma, PN: 46955) and $\mathrm{TiO}_{2}$ (Acros, $\left.\mathrm{PN}: 21358100\right)$ to yield $0.1 \mathrm{mM}$ fluorescein and $10 \mathrm{mg} / \mathrm{mL} \mathrm{TiO}_{2}$ in the agar solution. The addition of fluorescein creates a uniform fluorescent background in the phantom sample, while the addition of $\mathrm{TiO}_{2}$ serves as the scattering component corresponding to a scattering coefficient of $\sim 40 \mathrm{~cm}^{-1}$, approximately 3 times less scattering than normal epidermal layer of skin [22, 23]. The lower attenuation was used to ensure that a larger portion of the available $z$-range could be utilized, which increased the resolution in the $z$-direction. A piece of medical gauze was stained for 5 minutes in a $1 \mathrm{mM}$ Rhodamine-B-ITC (Sigma, PN: R6626) solution at room temperature. The excess staining solution was removed through rinsing with 1x PBS. The stained gauze was embedded in the agar gel solution to provide a localized signal in the red channel, and the solution was allowed to solidify, yielding the final phantom specimen.

2.4. Human Skin Samples. A human skin specimen was obtained as discarded tissue from breast reduction surgery at the Sahlgrenska University Hospital (University of Gothenburg, Gothenburg, Sweden). The specimen was cut into 1 $\times 1 \mathrm{~cm}^{2}$ pieces and stored at $-20^{\circ} \mathrm{C}$. Prior to the imaging experiment, the specimen was thawed and mounted onto a glass slide without further processing.

2.5. Experimental Signal-to-Background Ratio (SBR). Fluorescence signals from both the red and the green channels were used to calculate the experimental SBR. The pixel intensity of the red channel originates from a combination 
of the signals from the RBITC-stained fiber in the focus $\left(R_{f}\right)$ and the background fluorescence $\left(R_{b}\right)$. Similarly, the pixel intensity of the green channel can be defined as the combination of the signal from the fluorescein in the focal region $\left(G_{f}\right)$ and the background signal from the fluorescence evenly distributed in the gel $\left(G_{b}\right)$. The SBR should ideally be calculated as a ratio between the signals from the focus and the background as

$$
\operatorname{SBR}=\frac{R_{f}}{G_{b}}
$$

however, for practical reasons, the SBR was approximated by the ratio between the total signals in both channels as

$$
\mathrm{SBR} \approx \frac{R}{G}=\frac{R_{f}+R_{b}}{G_{f}+G_{b}} ;
$$

this simplification is based on the assumption that $R_{f} \gg$ $R_{b}$ and $G_{b} \gg G_{f}$. As seen from the experimental data, the $G_{f}$ component cannot be neglected. Taking this into consideration, this formula will give an underestimation of SBR and is thus considered a valid approach. The SBR were extracted from the different $z$-levels by extracting the mean fluorescent value of the total $x, y$ image. The data are presented as bar graphs with standard error.

\section{Results and Discussion}

3.1. Theoretical Considerations Exploring Annular Beams. In order to explore the intensity distribution around the focal volume when changing from Gaussian to annular illumination in MPM, the Fresnel-Kirchhoff integral (see (1)) was calculated on a grid around the focus using MATLAB for different beam ratios $b / B$. As shown in Figure 4 , the focal volume becomes elongated when the ratio $b / B$ gets closer to 1 , approaching a Bessel beam. This elongation can be utilized for other microscopy applications, for example, in two-photon excitation light sheet microscopy $[4,24]$, but for the current application, the elongation causes a reduction of the axial resolution.

To allow for Nyquist sampling at the cellular level, the focal volume should not exceed $2 \mu \mathrm{m}$ in the axial direction. From the data for the theoretical simulations (Figure 4), the full width at half maximum (FWHM) in the $z$-direction was extracted as a function of $b / B$, as presented in Figure 5. As demonstrated by the figure, FWHM values below $2 \mu \mathrm{m}$ are obtained when the $b / B$ ratio is kept below 0.6 . For ratios above this value, the FWHM rapidly increases the elongation of the focal volume above $2 \mu \mathrm{m}$. Thus, when implementing annular beams for MPM, $b / B$ should be kept below 0.6. As the $b / B$ ratio approaches 1 , the beam takes the shape of a Bessel beam with an infinitely long focal volume. The differentiation of the same value shows rapid elongation of the focal volume for $b / B$ values approaching 1 .

The potential generation of out-of-focus fluorescence was calculated for a range of different annular beams $(b / B=$ 0.2 to 0.95$)$ and compared to Gaussian illumination $(b / B=$ 0 ). The simulations were implemented by considering the
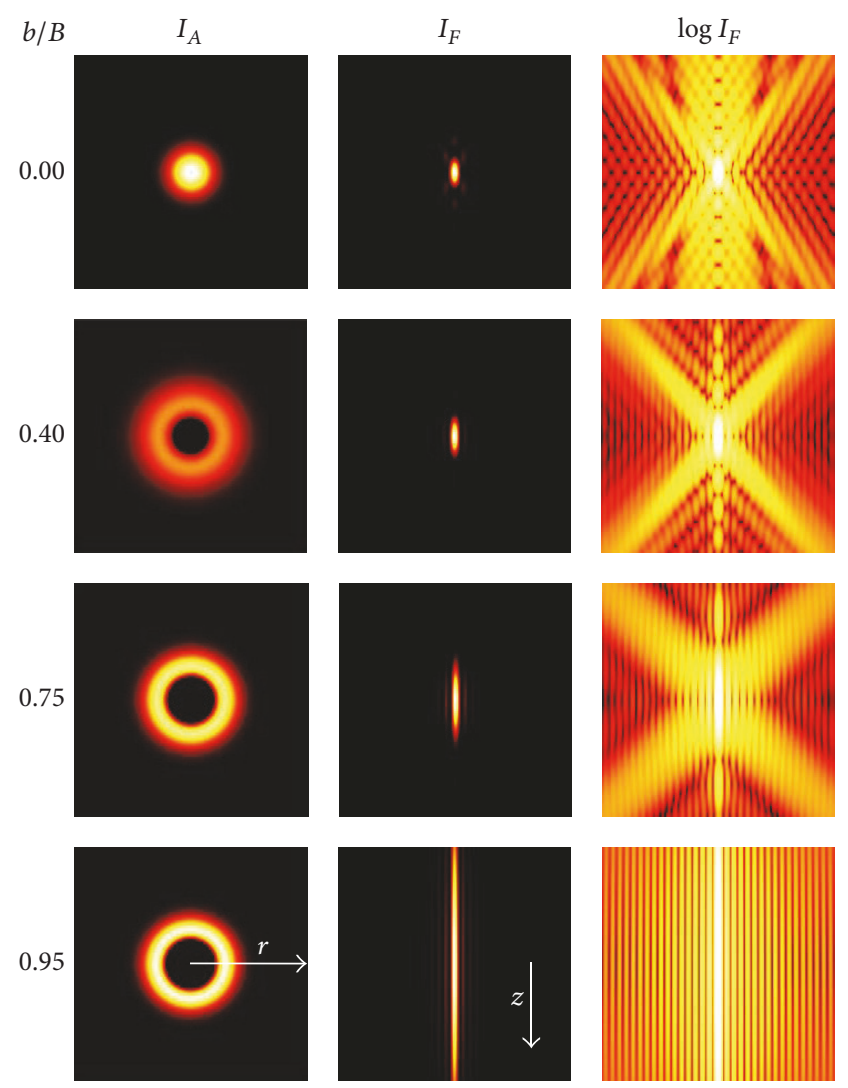

FIgURE 4: The distribution of intensity, proportional to the field amplitude squared as modeled by the Fresnel-Kirchhoff integral for different annular scenarios with varying $b / B$ using a maximum beam waist of $1.7 \mathrm{~mm}$ and focal distance of $2.7 \mathrm{~mm}$. $I_{A}$ is the input beam profile at aperture exit and $I_{F}$ is the simulated intensity distribution around the focal volume. Included is also $\log I_{F}$ to visualize the acquired diffraction pattern.

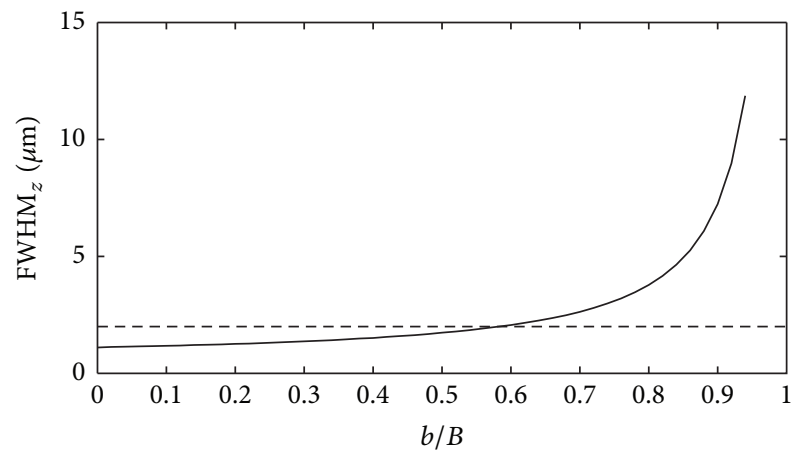

FIGURE 5: The calculated $\mathrm{FWHM}_{z}$ (solid line) along the $z$-axis as a function of $b / B$ obtained from the theoretical simulations for a 0.7 NA objective. To maintain a focal extent within twice the diffractionlimited performance, $\mathrm{FWHM}_{z}$ should be less than $2 \mu \mathrm{m}$ (dashed line). Thus, annular ratios should be kept $<0.6$ to avoid loss in $z$ resolution.

different geometrical distributions of the excitation light for the varying conditions. The results are presented in Figure 6 for two scenarios with different collection efficiencies (Gaussian and flat) assuming the scattering properties of 


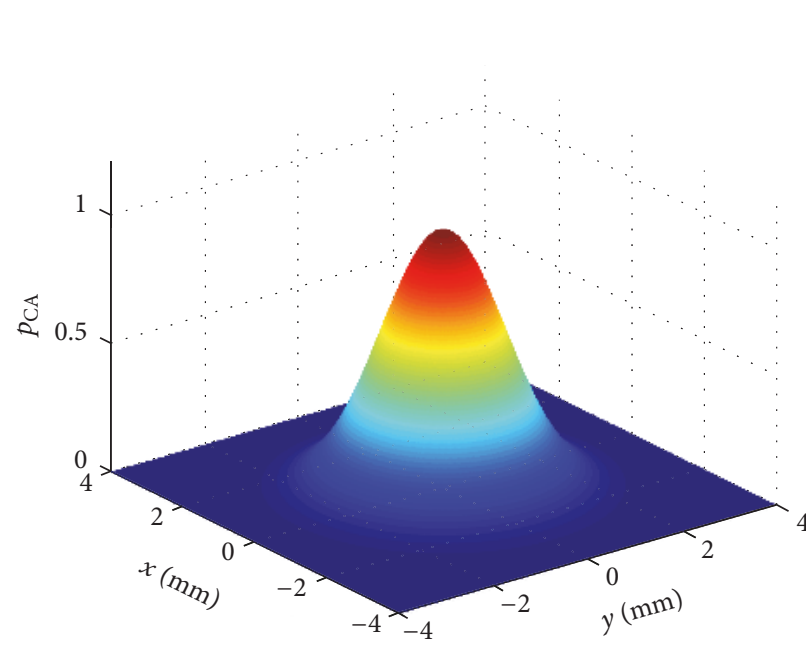

(a)

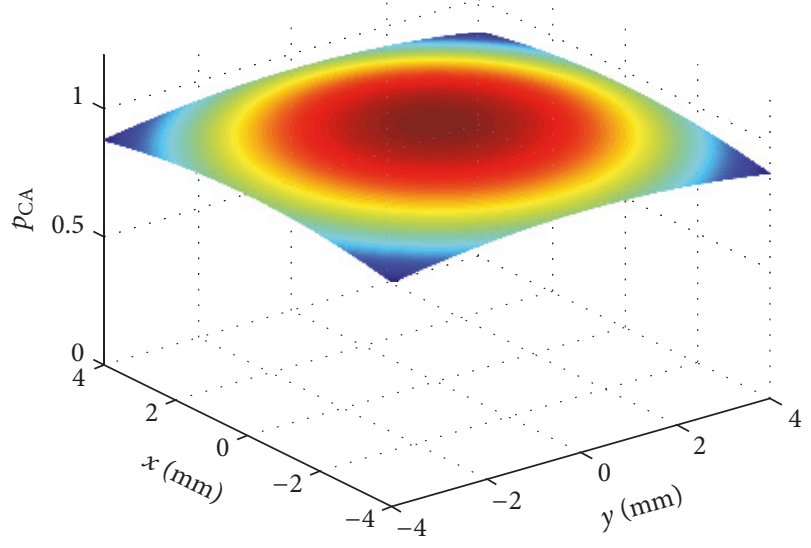

(c)

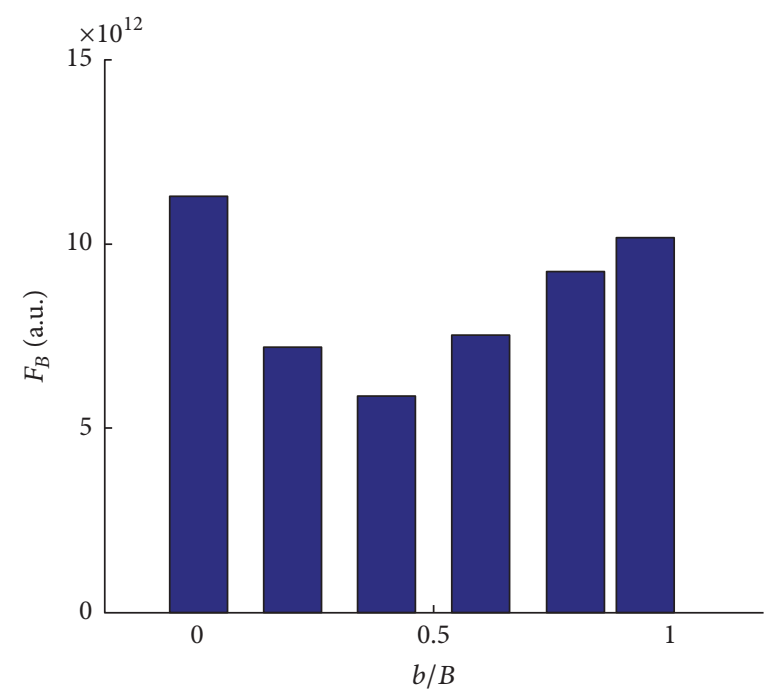

(b)

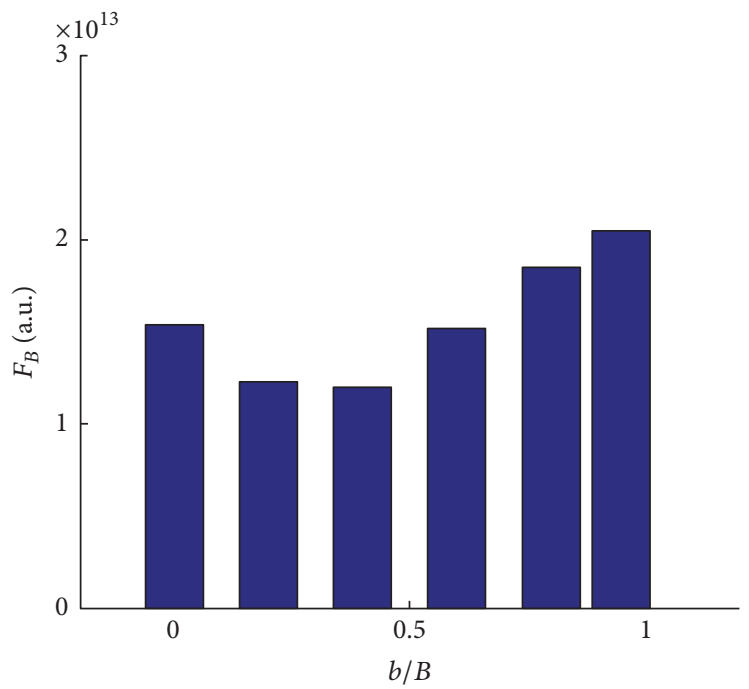

(d)

FIGURE 6: Results from the computer simulations estimating the out-of-focus signal $\left(F_{B}\right)$ generated for different shapes of the annular beam given as the ratio $b / B$. The presented simulations were performed using a mathematical approach considering the varying geometrical distribution of the excitation light depending on $b / B$. Results are presented for two scenarios assuming different probabilities of collection efficiency $\left(p_{\mathrm{CA}}\right)$ : (a) illustrating Gaussian and (c) flat collection efficiency. The respective results for simulated out-of-focus signal $F_{B}$ are presented in (b) and (d). For both cases, the value of the attenuation coefficient $\left(\mu_{s}\right)$ was set to $150 \mathrm{~cm}^{-1}$.

human skin. It can be seen from the figure that, in both cases, the out-of-focus fluorescence is expected to decrease to a minimum value for annular beams where the ratio of $b / B$ is kept around 0.4. For larger ratios, the generated out-of-focus fluorescence will increase. In the case of a more uniform collection efficiency across the objective, a too narrow annular beam will act contradictorily and elevate the probability of collecting out-of-focus signal (Figures 6(c) and 6(d)). In the case with a more realistic paraxial collection efficiency with the same waist as the objective exit aperture (Figures 6(a) and 6(b)), the background signal is reduced also for very narrow annulus. These computational simulations demonstrate that an annular beam with $b / B$ of around 0.4 should be optimal considering the tradeoff between reduction of out-of-focus signal and retaining the imaging resolution. In the simulations, the background signal can be reduced by half by switching from Gaussian to annular beam $(b / B=0.4)$, assuming paraxial collection efficiency.

3.2. Imaging Tissue Phantoms. In order to implement and experimentally explore how annular beams can reduce the out-of-focus signal in MPM, a wavefront controlling element, that is, an SLM, was included in the optical path of an experimental MPM setup, to allow for versatile control of the beam shape and simple switching between Gaussian and annular beam conditions. Annular beams were created 


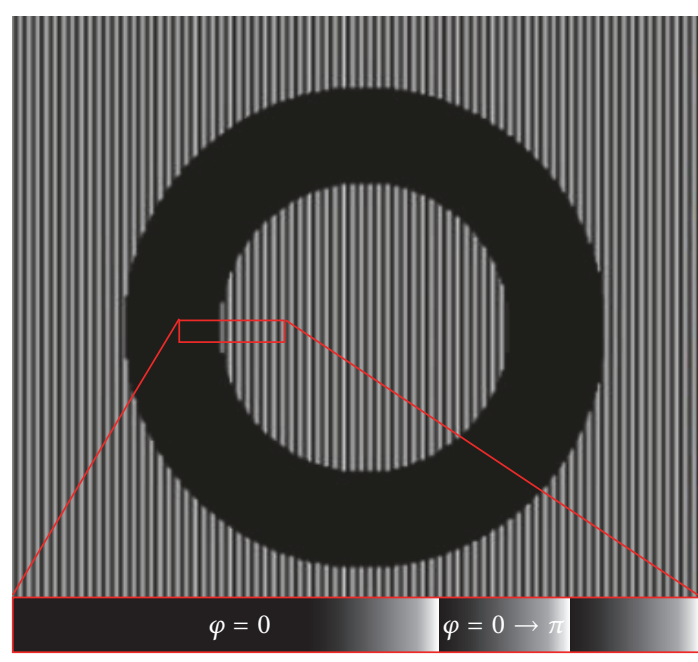

(a)

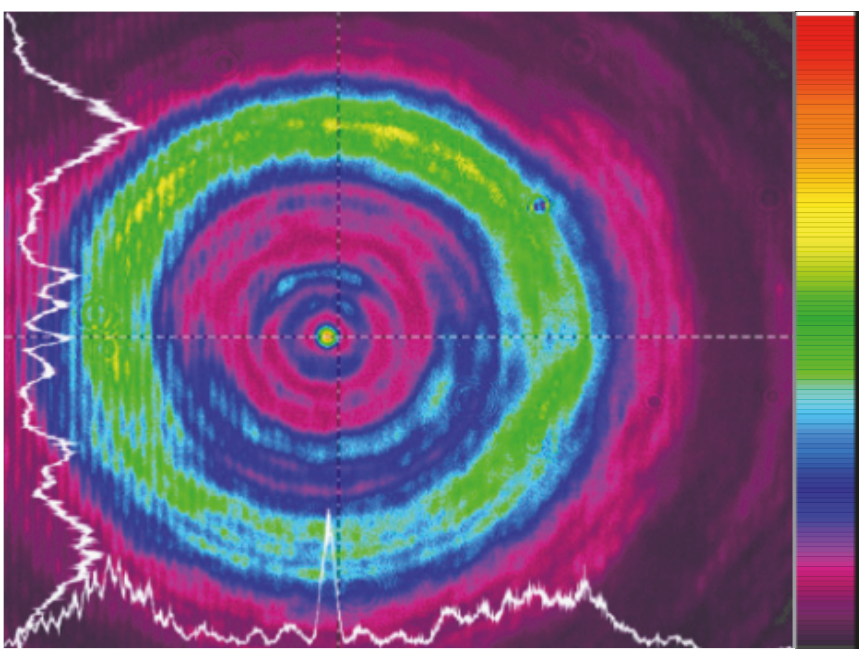

(b)

FIGURE 7: (a) The phase pattern applied at the SLM to generate an annular beam. The desired annulus is displayed in reflection mode with no phase shift $(\varphi=0)$ displayed in black, while the undesired part of the beam is deflected by applying a phase shift gradient $(\varphi=0 \rightarrow \pi)$. (b) A representative image of the acquired beam profile measured after the SLM. Dimensions of beam profiler image: $4.7 \mathrm{~mm} \times 6.3 \mathrm{~mm}$.

by diverting the central part of the beam uploading a phase pattern to the SLM. Figure 7 shows a typical phase pattern applied to the SLM and the corresponding generated beam profile obtained as measured in the beam path. As shown by the figure, the created annular beam is far from perfect, and future work will involve improvements of the generation of the annular beam to optimize the approach. For example, more efficient beam generation may be desirable, where the central part of the beam is not diverted and dumped to the side to avoid power loss. This is of particular importance for applications requiring deep tissue imaging, since high laser powers are needed to reach deep into the samples. Future efforts could adopt a Fourier plane setup, in which iterative Fourier transform algorithms [25] can be implemented to generate the desired annuli. Still, the generated beams using the simple diverting setup were sufficient in investigating the hypothesis experimentally. Therefore, the present study encourages future refinements of the approach.

According to the theoretical calculations, an annular beam with ratio $b / B$ of around 0.4 should be optimal in terms of maximum background signal reduction while keeping the axial resolution in a desired range. Thus, experimental efforts were made to generate an annular beam with these conditions, which were then implemented to image tissue simulating fluorescent scattering phantoms. The phantoms were created so that the RBITC-stained gauze fibers contribute to the in-focus signal (red channel), while the generation of outof-focus signal should appear most prominently as fluorescein fluorescence (green channel). Figure 8 shows data from a side-by-side comparison of representative MPM images of the tissue phantom acquired using annular versus Gaussian illumination with matching laser power. Both image data (at $\sim 550 \mu \mathrm{m}$ subsurface corresponding to 22 scattering lengths) and the extracted SBR values at different depths are presented in the figure. Similar structures are visualized using both Gaussian and annular beam illumination. As expected, the background signal is reduced when using the annular beam illumination, confirming the hypothesis. Also seen by the graph is that the improvement depends on the imaging depth. As expected, the improvement in SBR is small at shallow depths, but it increases at greater depths into the sample, with a maximal improvement of $30 \%$ at a depth of $550 \mu \mathrm{m}$. When imaging even deeper, the signal becomes limited, thus reducing the SBR. These results imply that annular beam is a feasible approach to reduce out-of-focus signal, as validated in these simplified tissue phantoms.

3.3. Proof of Principle in Tissue Sample. In order to explore the feasibility of annular beams for MPM in a more complex tissue sample, specimens of unstained excised human skin were examined ex vivo. Figure 9 shows MPM images of autofluorescent features of the dermal part of human skin at two different tissue depths acquired using both annular and Gaussian beam illumination. As shown by the figure, the fluorescence signal generated by the elastin and collagen fibers of the dermis is clearly visualized. At $45 \mu \mathrm{m}$ depth, the features observed are comparable using power-matched annular and Gaussian illumination. When imaging deeper into the dermis (around $80 \mu \mathrm{m}$ ), the imaging contrast for the Gaussian illumination is deteriorated as the signal from the fiber structures becomes blurry. For the annular illumination, the overall signal is reduced as expected, but the out-of-focus signal has been reduced. Even if the demonstrated effect at this stage in tissue is suboptimal, the experiments show proof of principle that (i) annular beams can be applied to perform MPM in complex biological tissue and (ii) the hypothesis of reducing out-of-focus fluorescence by annular beam shape 


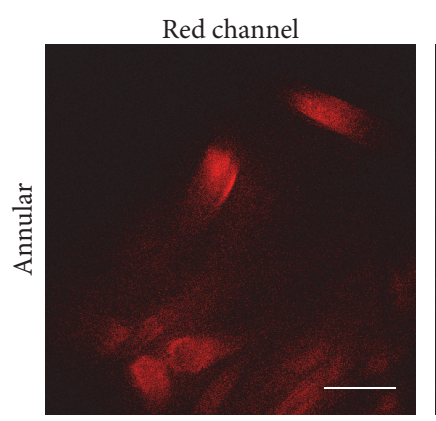

(a)

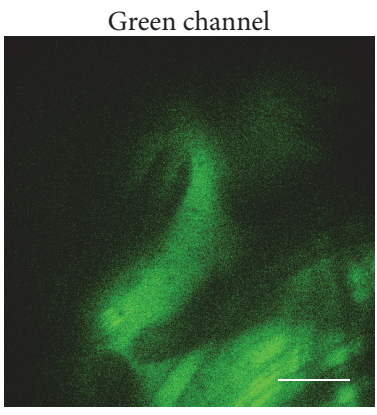

(a)
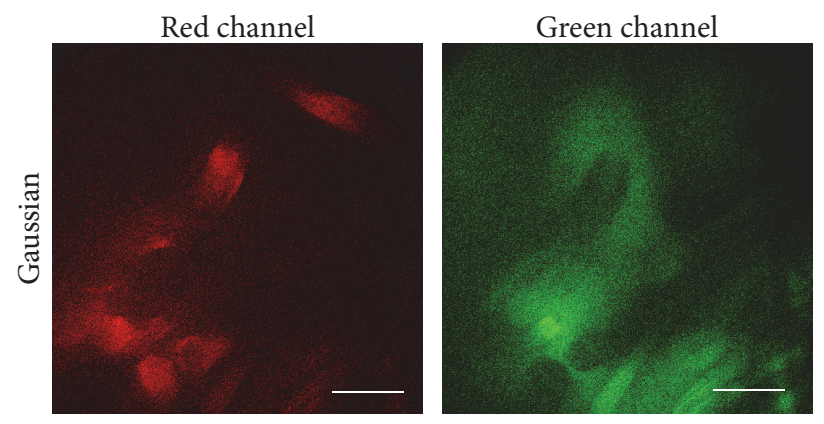

(b)

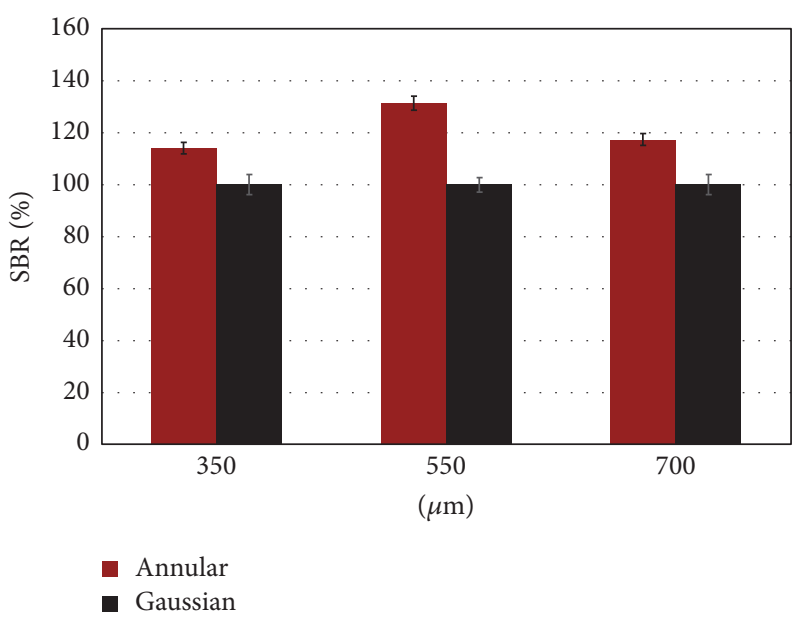

(c)

FiguRE 8: MPM images of a tissue mimicking phantom using power-matched ( $15 \mathrm{~mW}$ at the back aperture) annular (a) and Gaussian (b) beam illumination at $\sim 550 \mu \mathrm{m}$ subsurface. Field of view $\sim 250 \times 250 \mu \mathrm{m}^{2}$; (c) comparison of the signal-to-background ratio (SBR) extracted from MPM images at different imaging depths. The SBR is normalized to the Gaussian SBR at each imaging depth to show relative improvement in contrast. Each image was divided into 16 nonoverlapping regions of interest of identical size.

is confirmed. Future work should be undertaken to improve more efficient generation of the annular beam, particularly with respect to retaining laser power and accounting for dispersion effects.

\section{Conclusions}

This study explores annular beam shaping as a viable approach to decrease out-of-focus background fluorescence when performing MPM in optically turbid media. Based on the findings from computational simulations and mathematical models, the optimal regime for background reduction was found to be a beam ratio $b / B$ of around 0.4. Higher ratios degrade axial resolution while lower ratios do not eliminate significant out-of-focus background fluorescence. Imaging data from both tissue phantoms and excised tissue specimens demonstrate proof of principle and support feasibility and relevance in translational applications. Improved imaging contrast in annular illuminated MPM enables an increase in imaging depth, which can potentially allow for the monitoring of tumors and other pathological events in the microenvironment within the dermal layer of the skin, which is important in image-guided diagnostics [7] and pharmaceutical development [26]. The approach could potentially be combined with other approaches for improving imaging depths, for example, using third-harmonic generation [13, 27]. Additional quantitative imaging in light scattering tissue using techniques such as fluorescence correlation spectroscopy (FCS) and fluorescence lifetime microscopy (FLIM) would significantly benefit from reduced background. Improved imaging depth is also important to facilitate neuroscience [11] as it could potentially enable the noninvasive studies of layer $\mathrm{V}$ neurons and provide a better understanding of the neurocircuitry. Thus, future refinement and development are necessary in order to harvest full potential of the technique, which in turn could make MPM a more powerful, versatile, and indispensable tool in biomedical research.

\section{Conflicts of Interest}

The authors declare that there are no conflicts of interest regarding the publication of this paper. 

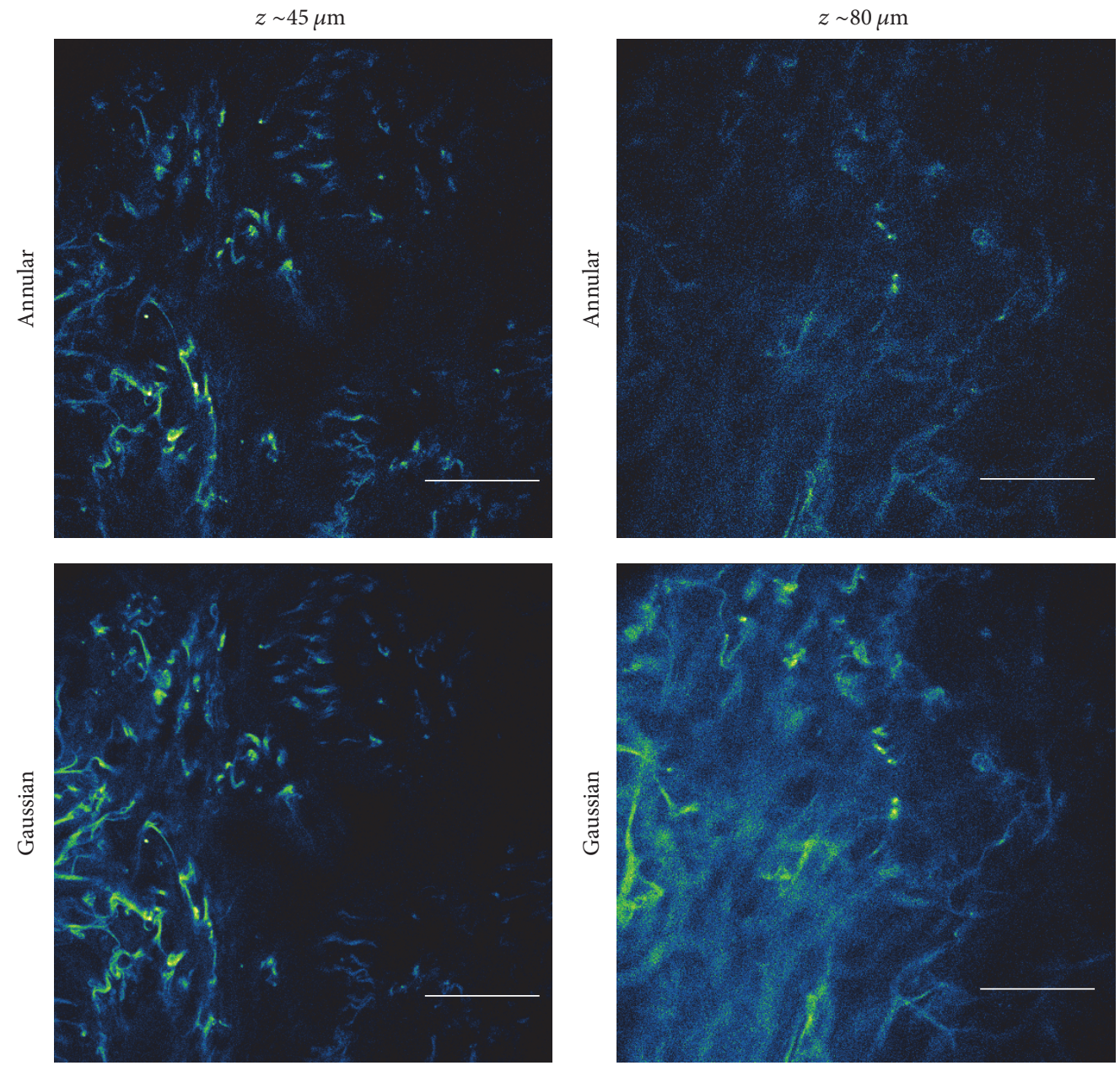

(a)

(b)

Figure 9: MPM images from the dermis of an excised unstained normal human skin specimen applying annular and Gaussian beam illumination at different tissue depths $(\sim 45 \mu \mathrm{m}$ and $\sim 80 \mu \mathrm{m})$ using matching laser powers $(\sim 15 \mathrm{~mW}$ and $20 \mathrm{~mW}$, resp. $)$ at the back aperture. Scale bar $=50 \mu \mathrm{m}$.

\section{Acknowledgments}

The authors acknowledge the staff from the Departments of Dermatology and Plastic Surgery at Sahlgrenska University Hospital for providing the excised skin specimens and the Centre for Skin Research (SkinResQU) for use of facilities. Financial support for this project was obtained from the Swedish Research Council (VR Dnr 621-2011-5189), Vinnova (VINNMER 2008-03414), and Lundberg Research Foundation (2013-430).

\section{References}

[1] W. Denk, J. H. Strickler, and W. W. Webb, "Two-photon laser scanning fluorescence microscopy," Science, vol. 248, no. 4951, pp. 73-76, 1990.

[2] W. R. Zipfel, R. M. Williams, and W. W. Webb, "Nonlinear magic: multiphoton microscopy in the biosciences," Nature Biotechnology, vol. 21, no. 11, pp. 1369-1377, 2003.
[3] C. Xu, W. Zipfel, J. B. Shear, R. M. Williams, and W. W. Webb, "Multiphoton fluorescence excitation: new spectral windows for biological nonlinear microscopy," Proceedings of the National Academy of Sciences of the United States of America, vol. 93, no. 20, pp. 10763-10768, 1996.

[4] T. A. Planchon, L. Gao, D. E. Milkie et al., "Rapid threedimensional isotropic imaging of living cells using Bessel beam plane illumination," Nature Methods, vol. 8, no. 5, pp. 417-423, 2011.

[5] J. Textor, A. Peixoto, S. E. Henrickson, M. Sinn, U. H. Von Andrian, and J. Westermann, "Defining the quantitative limits of intravital two-photon lymphocyte tracking," Proceedings of the National Academy of Sciences of the United States of America, vol. 108, no. 30, pp. 12401-12406, 2011.

[6] W. Supatto, A. McMahon, S. E. Fraser, and A. Stathopoulos, "Quantitative imaging of collective cell migration during Drosophila gastrulation: multiphoton microscopy and computational analysis," Nature Protocols, vol. 4, no. 10, pp. 1397-1412, 2009.

[7] J. Paoli, M. Smedh, A.-M. Wennberg, and M. B. Ericson, "Multiphoton laser scanning microscopy on non-melanoma 
skin cancer: morphologic features for future non-invasive diagnostics," Journal of Investigative Dermatology, vol. 128, no. 5, pp. 1248-1255, 2008.

[8] B. R. Masters, P. T. C. So, and E. Gratton, "Multiphoton excitation fluorescence microscopy and spectroscopy of in vivo human skin," Biophysical Journal, vol. 72, no. 6, pp. 2405-2412, 1997.

[9] K. König, "Clinical multiphoton tomography.," Journal of Biophotonics, vol. 1, no. 1, pp. 13-23, 2008.

[10] U. Olcese, G. Iurilli, and P. Medini, "Cellular and synaptic architecture of multisensory integration in the mouse neocortex," Neuron, vol. 79, no. 3, pp. 579-593, 2013.

[11] O. Garaschuk, R.-I. Milos, C. Grienberger, N. Marandi, H. Adelsberger, and A. Konnerth, "Optical monitoring of brain function in vivo: From neurons to networks," Pflugers Archiv European Journal of Physiology, vol. 453, no. 3, pp. 385-396, 2006.

[12] N. J. Durr, C. T. Weisspfennig, B. A. Holfeld, and A. Ben-Yakar, "Maximum imaging depth of two-photon autofluorescence microscopy in epithelial tissues," Journal of Biomedical Optics, vol. 16, no. 2, article 026008, 2011.

[13] D. Kobat, M. E. Durst, N. Nishimura, A. W. Wong, C. B. Schaffer, and $\mathrm{C} . \mathrm{Xu}$, "Deep tissue multiphoton microscopy using longer wavelength excitation," Optics Express, vol. 17, no. 16, pp. 1335413364, 2009.

[14] P. Theer and W. Denk, "On the fundamental imaging-depth limit in two-photon microscopy," Journal of the Optical Society of America A: Optics and Image Science, and Vision, vol. 23, no. 12, pp. 3139-3149, 2006.

[15] C. J. R. Sheppard, "Use of lenses with annular aperture in scanning optical microscopy," Optik, vol. 48, no. 3, pp. 329-334, 1977.

[16] S. W. Hell, P. E. Hänninen, A. Kuusisto, M. Schrader, and E. Soini, "Annular aperture two-photon excitation microscopy," Optics Communications, vol. 117, no. 1-2, pp. 20-24, 1995.

[17] C. J. R. Sheppard and A. Choudhury, "Annular pupils, radial polarization, and superresolution," Applied Optics, vol. 43, no. 22, pp. 4322-4327, 2004.

[18] J. Borglin, N. J. Durr, S. Guldbrand et al., "Improving multiphoton microscopy using annular beam shaping, focusing on imaging of human skin," in Proceedings of Multiphoton Microscopy in the Biomedical Sciences XIV, vol. 8948, SPIE, February 2014.

[19] Y. Nie, X. Li, J. Qi et al., "Hollow gaussian beam generated by beam shaping with phase-only liquid crystal spatial light modulator," Optics and Laser Technology, vol. 44, no. 2, pp. 384389, 2012.

[20] Y. Liu, P. He, and D. Cline, "Vacuum laser acceleration tests," in Proceedings of the 1999 Particle Accelerator Conference, vol. 5, pp. 3639-3641, 1999.

[21] I. V. Meglinski and S. J. Matcher, "Quantitative assessment of skin layers absorption and skin reflectance spectra simulation in the visible and near-infrared spectral regions," Physiological Measurement, vol. 23, no. 4, pp. 741-753, 2002.

[22] A. N. Bashkatov, E. A. Genina, V. I. Kochubey, and V. V. Tuchin, "Optical properties of human skin, subcutaneous and mucous tissues in the wavelength range from 400 to $2000 \mathrm{~nm}$," Journal of Physics D: Applied Physics, vol. 38, no. 15, pp. 2543-2555, 2005.

[23] E. Salomatina, B. Jiang, J. Novak, and A. N. Yaroslavsky, "Optical properties of normal and cancerous human skin in the visible and near-infrared spectral range," Journal of Biomedical Optics, vol. 11, no. 6, article 064026, 2006.
[24] F. O. Fahrbach, P. Simon, and A. Rohrbach, "Microscopy with self-reconstructing beams," Nature Photonics, vol. 4, no. 11, pp. 780-785, 2010.

[25] M. Walde, A. Jost, K. Wicker, and R. Heintzmann, "Engineering an achromatic Bessel beam using a phase-only spatial light modulator and an iterative fourier transformation algorithm," Optics Communications, vol. 383, pp. 64-68, 2017.

[26] V. Kirejev, S. Guldbrand, J. Borglin, C. Simonsson, and M. B. Ericson, "Multiphoton microscopy-a powerful tool in skin research and topical drug delivery science," Journal of Drug Delivery Science and Technology, vol. 22, no. 3, pp. 250-259, 2012.

[27] M. Yildirim, N. Durr, and A. Ben-Yakar, "Tripling the maximum imaging depth with third-harmonic generation microscopy," Journal of Biomedical Optics, vol. 20, no. 9, 2015. 

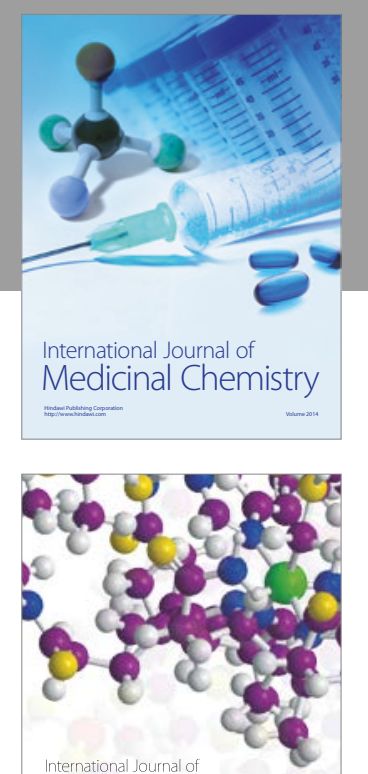

Carbohydrate Chemistry

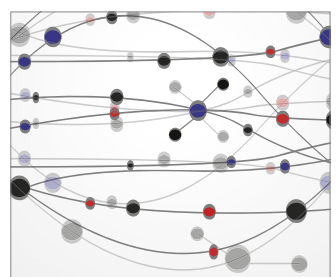

The Scientific World Journal
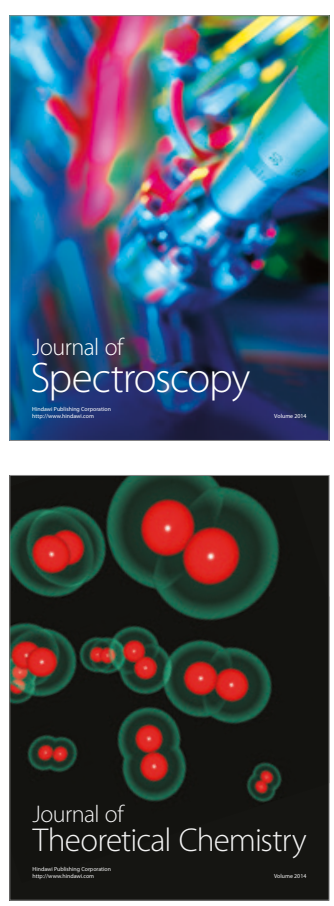
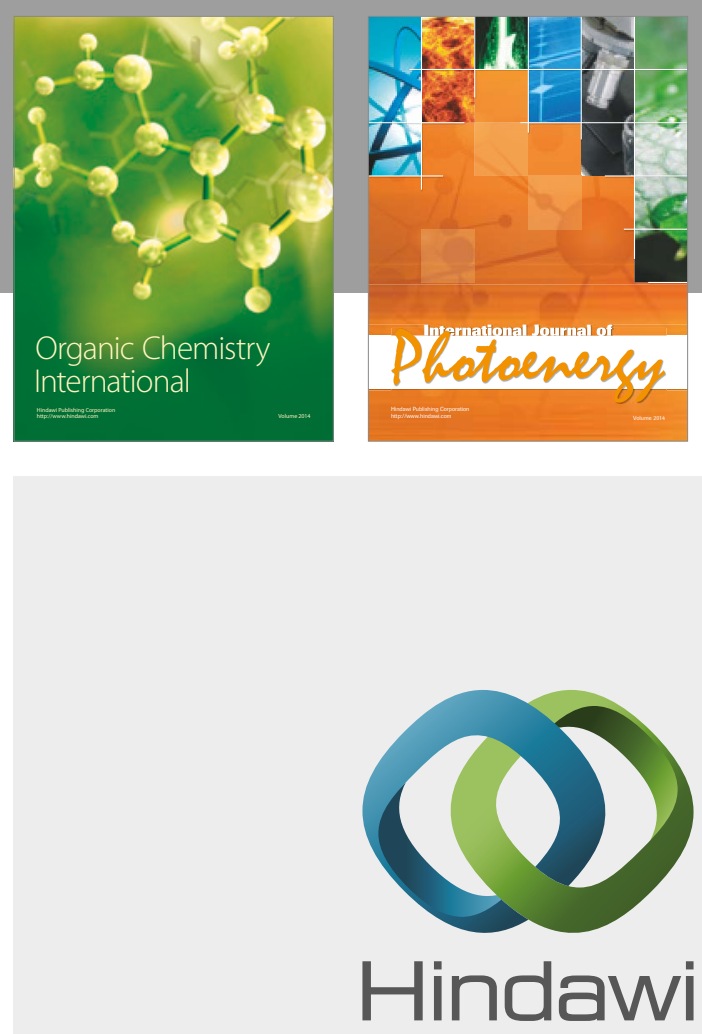

Submit your manuscripts at

https://www.hindawi.com

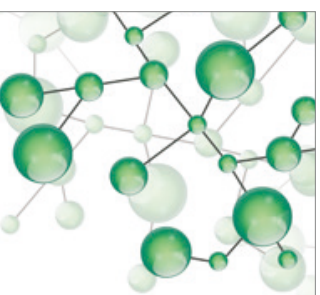

International Journal of

Inorganic Chemistry

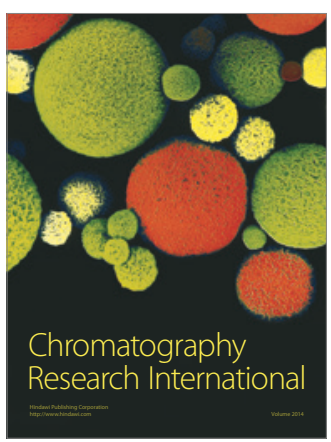

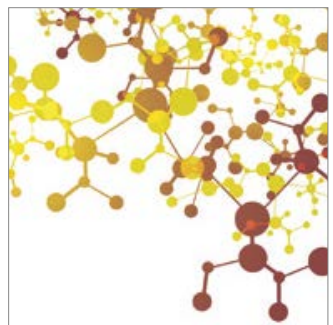

Applied Chemistry
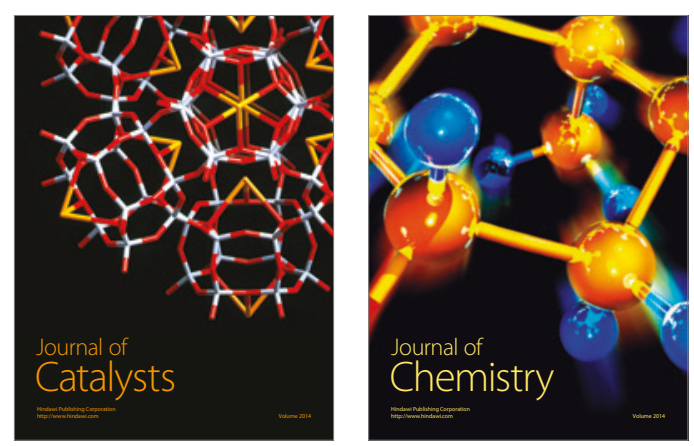
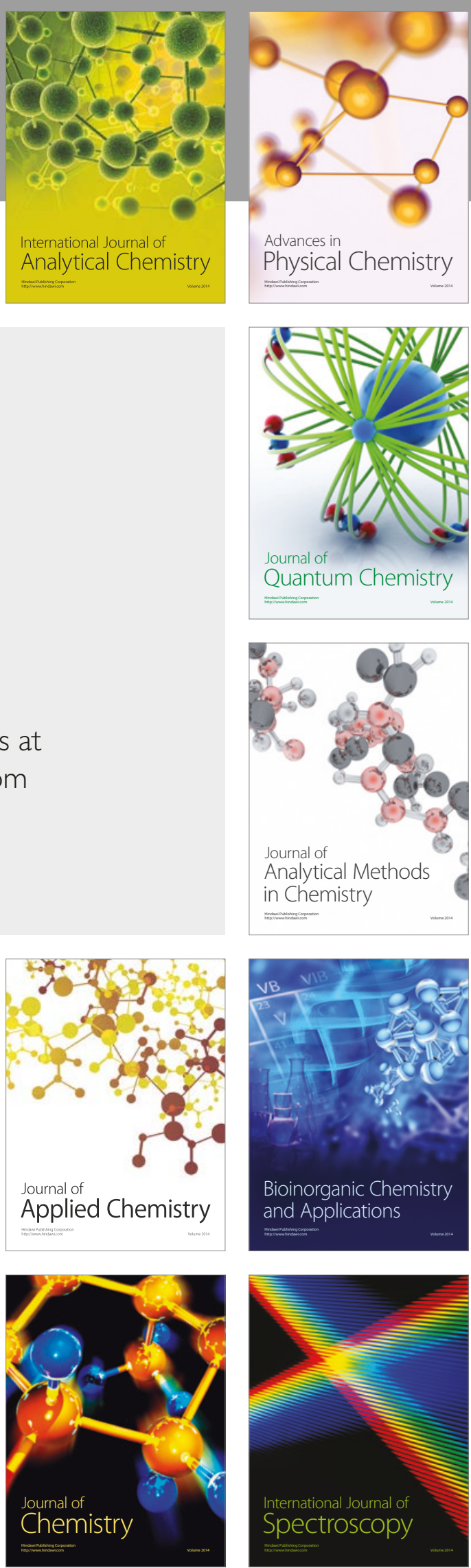